\title{
Mitigating the Spread of Measles under Constrained Health Care Resources in Tanzania using Social Contact Network Models
}

\author{
Herieth Rwezaura $^{1^{*}}$ and Seán McGarraghy ${ }^{2}$ \\ ${ }^{I}$ Department of Mathematics, University of Dar es Salaam, P.O. Box 35062, Dar es Salaam, \\ Tanzania.E-mail addresses: heriethrwezaula@yahoo.com; rwezaula.herieth@udsm.ac.tz. \\ ${ }^{2}$ UCD Lochlann Quinn School of Business, University College Dublin, Belfield, Dublin 4, \\ Ireland. E-mail: Sean.McGarraghy@ucd.ie \\ *Corresponding author
}

Received 22 Jul 2021, Revised 14 Oct 2021, Accepted 23 Oct 2021, Published Dec 2021

DOI: https://dx.doi.org/10.4314/tjs.v47i5.1

\begin{abstract}
Tanzania revaccinates individuals during measles outbreaks, despite having scant healthcare resources. We construct epidemiological models of measles spread by employing a hybrid of existing social contact networks models to develop SEIR simulation model. Using demographic and measles surveillance data from three rural villages in Tanzania, we simulate the spread of measles and examine which vaccination strategies can effectively control outbreaks. Results strongly indicate the spread of measles largely depends on contact rates among infected individuals within a population. Findings indicate a need for targeted vaccination for children of 6 months to 15 years of age, but equally for unvaccinated older age groups who were born before 1957 or missed the second dose. This work contributes theoretically and methodologically to existing applications of social contact network models for airborne infectious diseases in areas with health system constraints. It sets out implications for the design of effective vaccination programs for control of measles in Tanzania and in other developing countries.
\end{abstract}

Keywords: Vaccination strategies, Control strategies, Social contact network models, Airborne infectious diseases, Measles.

\section{Introduction}

Measles is one of the most contagious/transmissible airborne infectious diseases affecting susceptible individuals of all ages. It is a highly contagious febrile viral respiratory illness that can cause severe complications. Measles is one of the leading causes of death among young children globally (Goldrick 2019, WHO 2019, Berhe and Makinde 2020, Sowole et al. 2020). Despite availability of cheap, safe and high coverage of an effective single dose vaccine, large outbreaks continue to occur worldwide (Grais et al. 2007, WHO 2018, WHO 2020).

Measles outbreaks are normally centered in unvaccinated communities and have been linked to travelers from countries with ongoing measles outbreaks. In 2017, severe and protracted measles outbreaks caused a spike in measles cases globally due to gaps in vaccination coverage (WHO 2018). In 2019, measles cases surged worldwide to 869,770 , the highest number of reported cases in 23 years (Patel et al. 2020). Global measles deaths climbed nearly 50 percent from 2016 to 2019, claiming over 207,500 lives in 2019 (WHO 2020). The WHO (2021) notes that the number of reported cases is potentially underestimated as many infected individuals do not seek health care or if diagnosed, are either not totally or timely reported which 
could greatly impact timely informed decision.

Despite Tanzania's limited financial resources, revaccination of individuals continues during measles outbreaks (Mnyika and Akim 2005). Routine measles vaccination for all children at 9 months was introduced in 1975 as part of its Expanded Program on Immunization (Mandara and Remme 1983, Goodson et al. 2009). From 1995 to 2005, the responsible Ministry of Health ran annual mass immunization week campaigns involving all children under the age of five against all major childhood immunizable diseases including measles. In 2001, the age group eligibility for measles vaccination was extended to include children up to 15 years of age (Mnyika and Akim 2005). Measles first dose vaccination coverage increased from $46 \%$ in 1980 to $80 \%$ in 1990, fluctuating between $72 \%$ and $83 \%$ through the $1990 \mathrm{~s}$, and increased from $78 \%$ in 2000 to $>90 \%$ in 2003-2007 (WHO 2008). However, despite seemingly high vaccination coverage, measles is still re-occurring worldwide. Figure 1 shows the yearly distribution of measles cases in Tanzania from 2006 to 2021, while Figure 2 illustrates number of measles cases and deaths (from 2000-2010) in Nkasi District, which is the geographical area of interest for this paper.

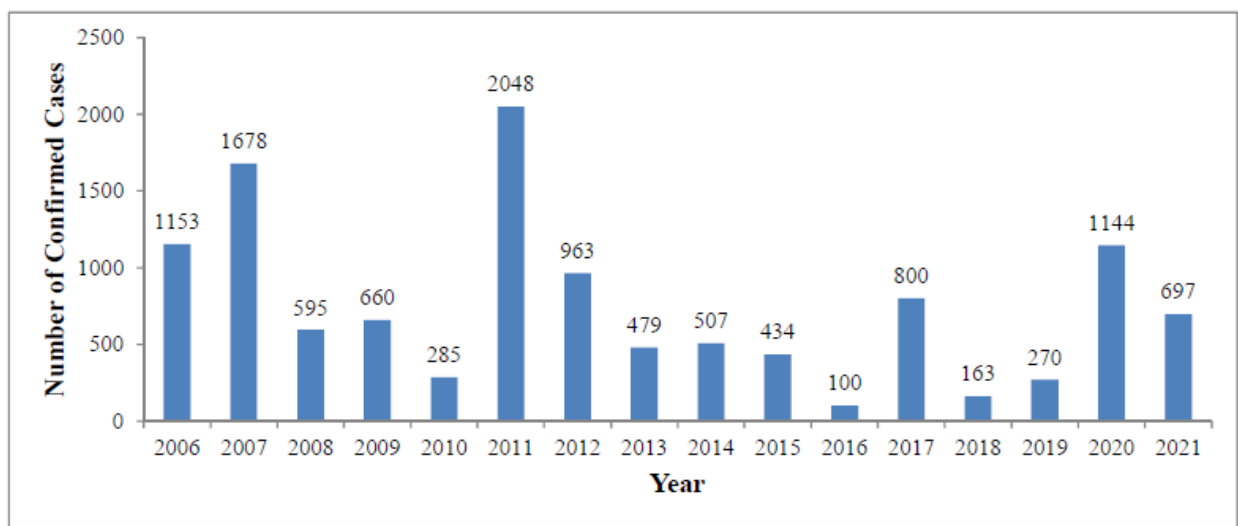

Figure 1: Distribution of measles cases in Tanzania from 2006 to 2021.

Source: WHO (2021).

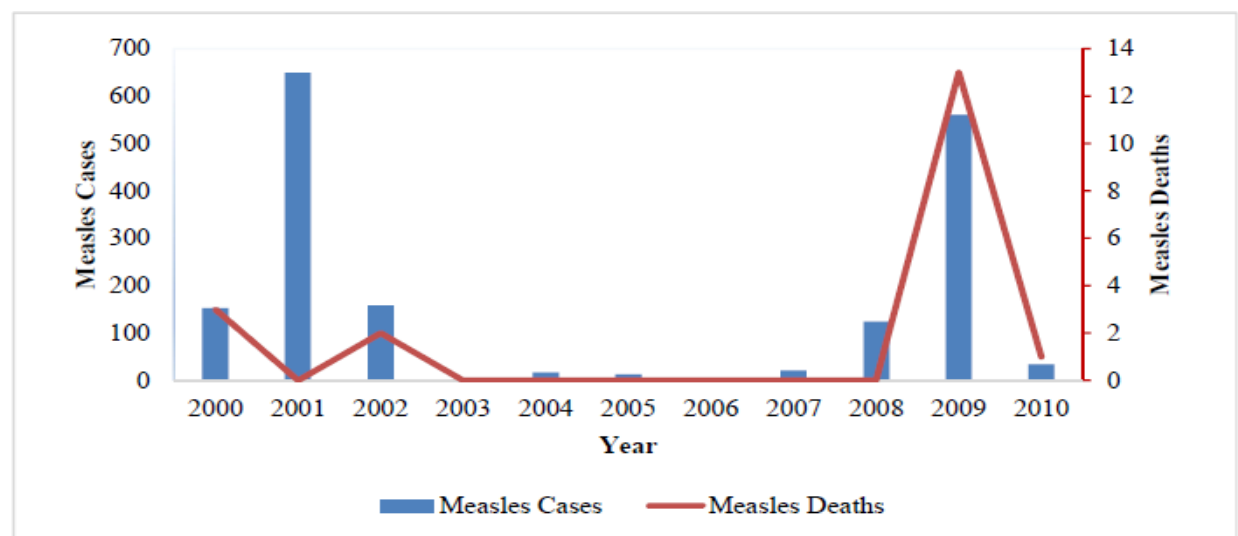

Figure 2: Number of measles cases and death in Nkasi District (2000-2010).

Source: Office of District Medical Officer, Nkasi District.

Due to the high burdens on countries' health care systems, the ongoing COVID-19 pandemic is expected to result in decline in immunization coverage, hence leading to 
increased risk of measles outbreaks (WHO 2020). Health infrastructure deficiencies in Tanzania hinder efforts to determine and implement effective prevention and control strategies for airborne infectious diseases. Nevertheless, public health policy and decision makers need reliable and consistent estimates of the impact of such diseases and the likely consequences of their interventions.

The social contact network, and the way individuals interact within, is critical to model the spreading dynamics of airborne infectious diseases (Newman 2002, May and Lloyd 2001, Meyers et al. 2003, Meyers et al. 2005). The approach has also been useful in examining the efficacy of various intervention strategies and in developing optimal control strategies.

It is important to note that vaccination is a major control measure to mitigate the spread of infectious diseases such as measles. It reduces individuals' susceptibility and their probability of infecting others (Martín et al. 2011). Pourbohloul et al. (2005) noted that the success of vaccinating a proportion of the population at random depends on the vaccination coverage, vaccine efficacy, and disease transmissibility, and that the availability of a vaccine, therefore, does not guarantee prevention unless both delivery and vaccine-induced immunity are sufficient. Efforts to find optimal (improved) vaccine interventions and implementation to curb the propagation of disease are of crucial in the context of constrained health care resources, like that of Tanzania.

We formulate a simulation model of social contact networks of individuals, in order to identify mechanisms that are significant for understanding the spread of measles and propose potential effective control strategies. We use demographic and measles surveillance data from three rural villages, namely Mkole, Korongwe and Mkinga in Nkasi District, Rukwa region in Tanzania in constructing and simulating social contact networks of individuals. Nkasi District has been facing measles outbreaks and the rampant one occurred in 2009, which caused social and economic burdens at the households, district, regional and national levels. Although the impact of measles in these villages is largely noted on the younger ages (children) compared to other age groups, our model is inclusive of adults because of mechanisms and patterns of interactions that exist between individuals of all age groups.

We use our social contact network epidemiology model to examine which vaccination strategies can effectively protect populations against measles in villages. Vaccination strategies are evaluated in the same with the structure of the population (age, number, and size of households). We use a SEIR (Susceptible, Exposed, Infected, and Recovered) model that incorporates vaccination of a proportion of the population before the outbreak of measles or at any other point during the outbreak.

We extend the standard social contact network models of airborne infectious diseases by incorporating aspects of the scale-free networks (the power-law degree distribution) created by preferential attachment (Barabàsi and Albert 1999). The choice of this type of hybrid social network is informed by its ability to mimic dynamical mechanism present in real-world networks.

While several studies have been conducted on measles' control strategies in Tanzania (Mandara and Remme 1983, Lyamuya et al. 1999, Kamugisha et al. 2003, Mnyika and Akim 2005, Goodson et al. 2009), none of these studies employed social contact network epidemiology. To the best of the knowledge of authors, the novelty of this paper lies in using a hybrid of existing networks models to develop reliable measles outbreaks predictions, simulate a social contact network of individuals, thereby offering a valuable contribution towards proposing optimal and/or improved control strategies for measles outbreaks. Specifically, this paper seeks to accomplish two aims; one, to develop a social contact network model that allows prediction of measles dynamics and; two, to develop a vaccination model using the standard SEIR-type model and eventually propose an optimal and/or improved control strategy. 


\section{Materials and Methods}

We construct a contact network model that captures the interactions that underlie respiratory disease transmission within Mkinga, Korongwe and Mkole villages. The demographic data for these villages are used to populate the network model. For each village, we use the age cohorts of individuals from ages 0 to $65+$ years from the 2002 Tanzania Population Census and the Nkasi District Population Projection (2010-2025). A total of 22,468 individuals are obtained. Figure 3 summarizes age distribution of the individuals in the three villages.

Individuals in these villages are assigned ages according to the Nkasi District's population projection and based on their age distribution they are assigned to different mixing groups or locations. School-going age children for instance are assigned to preschools/primary schools/secondary schools according to schools and class size distributions data. The remaining individuals are assigned to locations such as workplace and community (recreational centers, public venues, etc.) according to available information about the socio-economic activities in the villages. Thus, every individual in the community belongs to 1 of 7 age-dependent categories: infants (less than 1 year), toddlers (ages 1-4), preschool children (ages 5-6), primary school children (ages 7-9 and 10-14, respectively), secondary school children (ages 15-19) and adults (ages 20$65+$ ). Each of these individuals belongs to one or more mixing groups, namely, households, schools, workplaces, and the community. Interactions among individuals reflect household size school and other mixing group data for these villages.

To understand the patterns of the disease spread on individuals' contact networks, we estimate the daily time of contact of different age groups. We use the Country-Wide Time Use survey in Tanzania by Joint the Norwegian Council for Higher Education's Programme for Development Research and Education (NUFU) Project (2006) and the Value of Time in Least Developed Countries Report by Department for International Development (2005) to estimate the mean daily time.

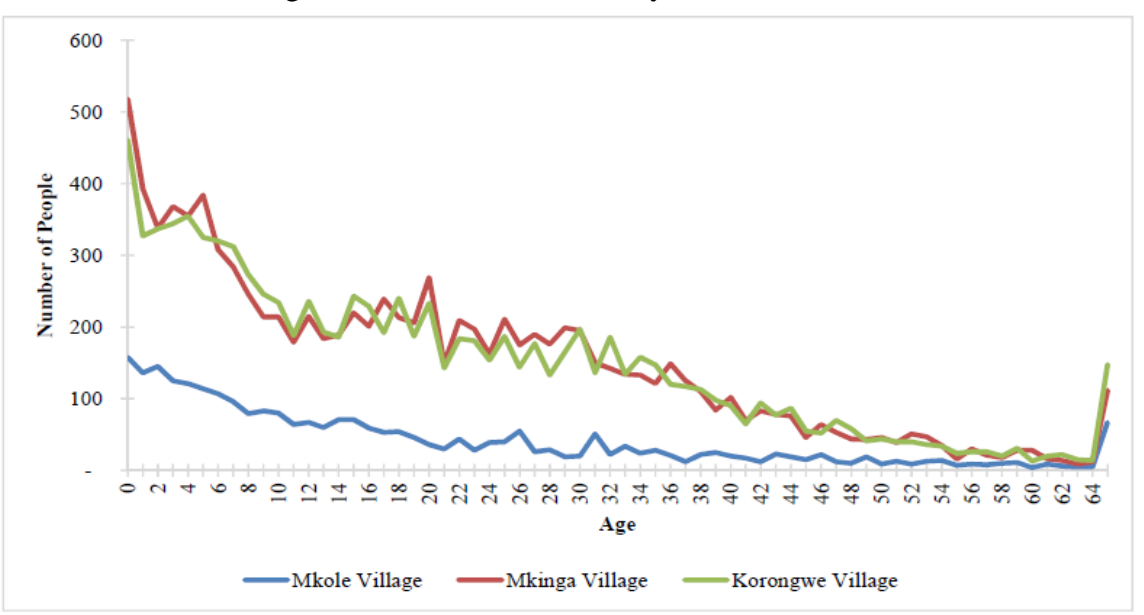

Figure 3: Age distribution of individuals in Korongwe, Mkinga and Mkole. Source: United Republic of Tanzania (2002).

We supplement the same with information from Burke and Beegle (2004), Kondylis and Manacorda (2006), Andvig et al. (2001) and Adhvaryu and Nyshadham (2011), as well as from rural dwellers narratives in the three villages. A fuller description of the model parameter values estimation can be found in the supplementary materials in Haber et al. (2007).

In generating the networks, individuals in a population are represented as vertices (nodes), while contacts between them that can possibly lead to disease spread are indicated by edges. A contact is considered as 
being present in the same transmission unit (e.g., household), which is relevant for the spread of airborne infections (measles in particular). The contact network begins with an initial connected network of $m_{0}$ nodes.

New nodes are added to the network one at a time. This contact network is created by preferential attachment as described by Barabàsi and Albert (1999), giving rise to power-law degree distribution.

\section{Simulation model}

We simulate measles outbreak in the three villages and the simulation is executed with a contact network and age-structure using MATLAB. To comprehensively simulate our model, we assume that people are at risk of getting measles if they have not been immunized or have been insufficiently immunized or have not been previously infected with measles. At the beginning of the simulation, the proportion of the population with measles immunity whether conferred through immunization or due to past infection is entirely removed from the network (because of its inability to infect or to be infected) and the rest of the population $(N)$ is assumed to be susceptible prior to introducing an infectious agent. Based on outlined assumptions by the Measles Strategic Planning and the Immunity Profile (by age) for measles in Tanzania, we assumed $50 \%$ of $<1$ year, $75 \%$ of $1-4$ years, $75 \%$ of 5-6 years, $85 \%$ of $7-9$ years, $90 \%$ of $10-14$ years, $95 \%$ of $15-19$ years and $98 \%$ of 20-65+ years to be the proportions of the population with measles immunity.

To estimate the probability of disease transmission, $r_{i j}$ (per day) our procedure follows that of Del Valle et al. (2007) and significantly draws from those in the Appendix in Haber et al. (2007). As in Haber et al. (ibid.), our model assumes that on any given day, a susceptible individual $i$ come into contact with other individuals that may lead him or her to become infected. The contacts take place in each of $i$ 's mixing groups. The probability that individual $i$ becomes infected depends on the following input parameters: (a) The number of different individuals with whom susceptible individual $i$ has contact in each mixing group.

(b) The total duration in hours, of all the contacts with each of these individuals, and

(c) The per-hour rates of infection transmission if the contacted individual is infectious.

\section{The SEIR model}

A standard SEIR-type model is used to simulate the measles outbreak. Susceptible individuals exposed to measles move to the infected but not infectious class, and after a given latent period some of these individuals become infectious until they either recover (and acquire long-lasting immunity after infection) or die. From Anderson and May (1991), measles' latent period is 6-9 days, and its infectious period is 6-7 days. For our case, we consider mean the latent period to be 8 days and the mean infectious period to be 7 days.

We consider it imperative to model both an outbreak that starts with a single-infected individual (vertex or node), and with multiple infected individuals (vertices or nodes) in the network. At each subsequent time step, a susceptible individual $i$ in contact with $I$ infectious individuals (vertices) may contract the disease during a period $\delta t$ with probability $P_{S E}=T_{i j}=1-e^{-r_{i j} I \delta t}$,

where $r_{i j}$ is the rate of transmission across an edge (i.e., the probability of disease transmission-per day), and $I$ is the number of infected individuals to which the individual $i$ is connected (Chowell-Puente and Sanchez 2001). Susceptible individual $i$ enters the exposed disease state (with probability $P_{S E}$ ) when he or she becomes infected but is not yet infectious. The exposed individual becomes infectious at a rate $\gamma$ (with probability $P_{E I}$ ), where $\gamma^{-1}$ represents the mean latent period of the disease. It is assumed that the latent period is exponentially distributed, i.e., the probability of infectivity in time interval $(t, t+\delta t)$ is given by $P_{E I}=1-e^{-\gamma \delta t}$.

Infectious individuals can transmit the disease during their infectious period with 
mean duration $\tau^{-1}$. It is also assumed that the infectious period is exponentially distributed, i.e., the probability of recovery $\left(P_{I R}\right)$ in the time interval $(t, t+\delta t)$ is given by

$P_{I R}=1-e^{-\tau \delta t}$.

After the mean infectious period of duration $\tau^{-1}$, infectious individual enters the removed state (i.e., recovered at a rate $\tau$ with life-long immunity or die). The process is repeated until the agent becomes extinct.

\section{The SEIR vaccination model and assumptions}

In our SEIR simulation model of vaccine interventions, we assume that vaccination grants partial protection to vaccinated individuals since vaccine is not $100 \%$ effective. This reflects to what Orenstein et al. (1985) noted, that under ideal circumstances, the measles vaccine has $85 \%$ efficacy when administered to children at nine months of age. However, the cold vaccine supply chain is particularly vulnerable in hot, rural areas in developing countries (such as Tanzania), where infrastructure is poor and monitoring may be inadequate (Talley and Salama 2003).

We consider vaccine efficacy as the reduction (after vaccination), in the probability of becoming infected due to contact with an infected person, or as the reduction (after vaccination), in the probability of infecting a susceptible contact (Andradóttir et al. 2011). In this vein, vaccine efficacy does not refer to the fraction of individuals having an immunogenic response to the vaccine. Our SEIR vaccination model incorporates the fact that vaccination reduces the transmission probability of the disease (the probability of acquiring an infection conditional on contacting an infective): an unvaccinated susceptible individual becomes infected with probability $r_{i j} \in(0,1]$ after contacting an infected individual, while the corresponding probability for a vaccinated susceptible individual is $r_{i j}(1-\varepsilon)$, where $\varepsilon \in(0,1]$ denotes the efficacy of the vaccine (Chen and Cottrell 2009).

At each subsequent time step $\delta t$, all individuals, infected or not, establish contact with another randomly chosen individual.
This means that an unvaccinated susceptible individual $i$ in contact with $I$ infectious individuals has the probability $T_{i j}=1-$ $e^{-r_{i j} I \delta t}$ of becoming infected at that time step $\delta t$, while the probability of acquiring an infection during the same time step is $T_{i j}=1-e^{-r_{i j}(1-\varepsilon) I \delta t}$ for a susceptible individual who has been vaccinated, where $r_{i j}$ is the rate of transmission across an edge (i.e., the probability of disease transmissionper day), $I$ is the number of infected individuals to which the individual $i$ is connected and $\varepsilon$ is the efficacy of the vaccine.

In identifying the most effective intervention(s) to control the outbreak of airborne infectious diseases, the efficacy of various vaccination strategies $v_{i}$ are assessed by reducing the probability of a vaccinated edge to be infected. Vaccination strategies that are applied in the epidemic model are: (i)Mass vaccination strategy where all susceptible individuals represented in the network by vertices are vaccinated; (ii) Targeted vaccination strategy where specific groups of individuals represented in the network by vertices based on risk factors such as age, health, and places of employment (or vaccinating vertices on places where risks of infection are highest) are vaccinated. Other vaccination strategies such as ring and random vaccination strategies are not considered herein. The above vaccination strategies $v_{i}$ are compared to obtain an optimal (improved) one that can control the outbreak on contact networks subject to real world constraints.

The following assumptions from the Measles Strategic Planning (Advanced Immunization Management 2009) are considered:

i. Measles immunity, whether conferred through immunization or infection is lifelong.

ii. Maternal antibodies provide protection against measles until 6 months of age.

iii. Vaccination provides partial protection to vaccinated individuals but if administered at regular intervals it provides an effective protection. 


\section{Results and Discussion}

This part presents and discusses results of both measles outbreak occurring at a single individual (vertex or node), and at multiple individuals (vertices or nodes) in the network, based on the SEIR simulation model for the three villages. To understand the fundamental topological properties of the underlying scale-free contact networks in these villages, we first present the degree distributions.

\section{Degree distribution and scale free networks}

The patterns by which epidemics spread through groups of people are determined not just by the characteristics of its infectious agents, such as its contagiousness, the length of its infectious period, and its severity, but also by the network structures within the affected populations (Easley and Kleinberg 2010). The structure of scale-free contact networks of these three villages would help to reflect the likelihood of measles spread from one contact to another as shown in Figures $4 a, 4 b, 5 a$ and $5 b$.

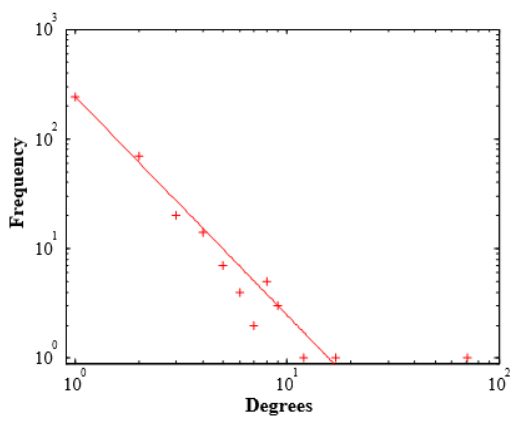

Figure 4a: Mkole Village: Degree distribution; $N=373$.

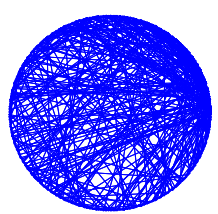

Figure 4b: Mkole Village Scale-free Network with $N=373$ and $m_{0}=3$. Nodes with higher degrees are close to each other and those with lower degrees are near to each other.

For illustration purposes, the network of individuals in Mkole and Mkinga villages, whose interactions take place in households and schools are constructed and plotted. Korongwe village was intentionally left in this illustration because its features are quite similar to that of Mkinga. The same number of households (13) was used and because the household sizes in Mkole and Mkinga differ, the total numbers of individuals in the network also differ. Mkinga village network appears dense because the village has more individuals in the households compared to Mkole village. Figure $4 \mathrm{c}$ shows the network of 91 individuals from Mkole village.

Figure $5 \mathrm{c}$ illustrates the plot of Mkinga network, a village network of 123 individuals from the same 13 numbers of households as in Mkole.

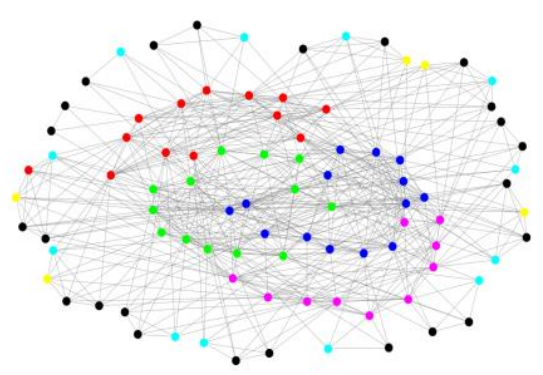

Key:

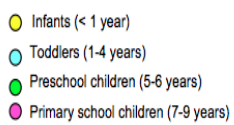

Primary school children (10-14 years) O Secondary school children (15-19 years) - Adults (20-65+years)

Figure 4c: Mkole Village Network $(N=91$; Households $=13$ ). Nodes with higher degrees are placed centrally and those with lower degree away from the centre. 


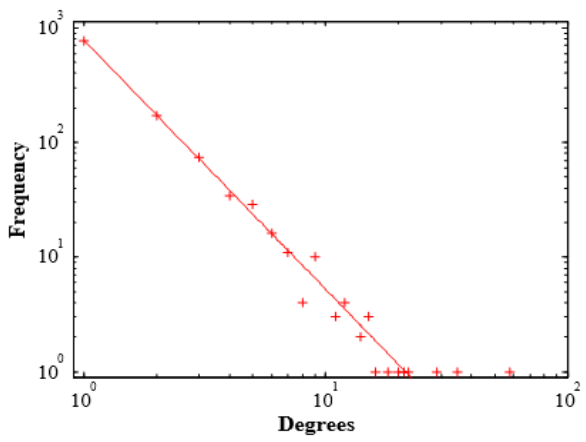

Figure 5a: Mkinga Village: Degree distribution; $N=1148$.

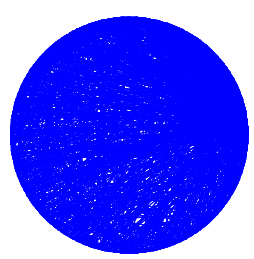

Figure 5b: Mkinga Village Scale-free Network with $N=1148$ and $m_{0}=3$. Nodes with higher degrees are close to each other and those with lower degrees are near to each other.

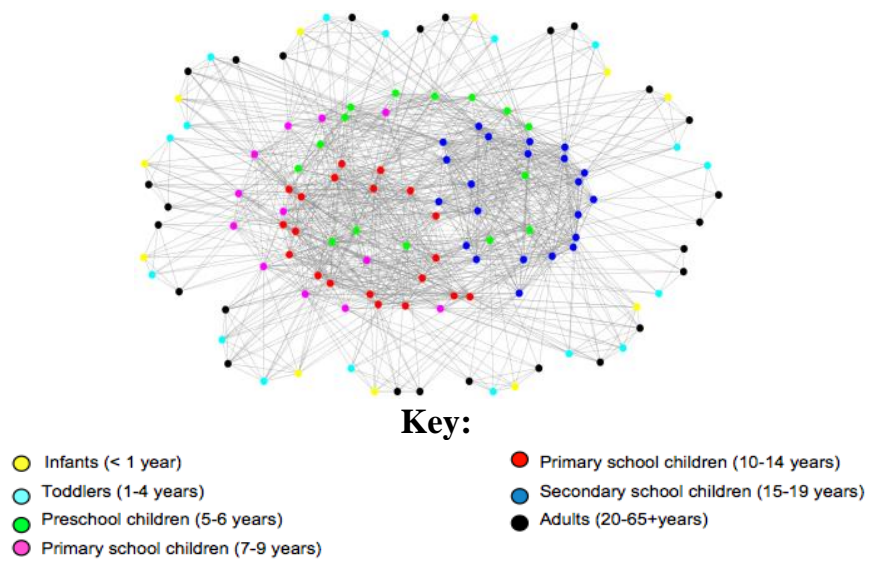

Figure 5c: Mkinga Village Network $(N=123$; Households $=13)$. Nodes with higher degrees are placed centrally and those with lower degree away from the centre.

\section{The SEIR epidemic simulation}

This part presents results of the SEIR epidemic simulation model of the scale free networks for Mkole, Mkinga and Korongwe villages. We consider the following three output measures: peak time, peak value and diffusion fraction (Rahmandad 2004). We included the uncertainty of the model in the second panels in Figures 7-8, represented by the shadows around each model variable. Rahmandad (2004) defined the peak time $T_{p}$ as the time from the introduction of infected individual zero to the maximum of the infected population, and it measures how quickly the epidemic spreads, and therefore how long officials and other personnel in public health must deploy control strategies. The peak value $I_{\max }$ of the symptomatic infected population indicates the peak load on the public health infrastructure for instance health workers, immunization resources and other health care facilities. Finally, the diffusion fraction, $F=R_{\infty} / N$ is the fraction of the initial population that ultimately is infected, and it measures the total burden of morbidity and mortality borne by the population. Figure 6 shows a sample simulation showing four discrete states (different population groups) and defines three output measures, Diffusion fraction, Peak time, and Peak value. 


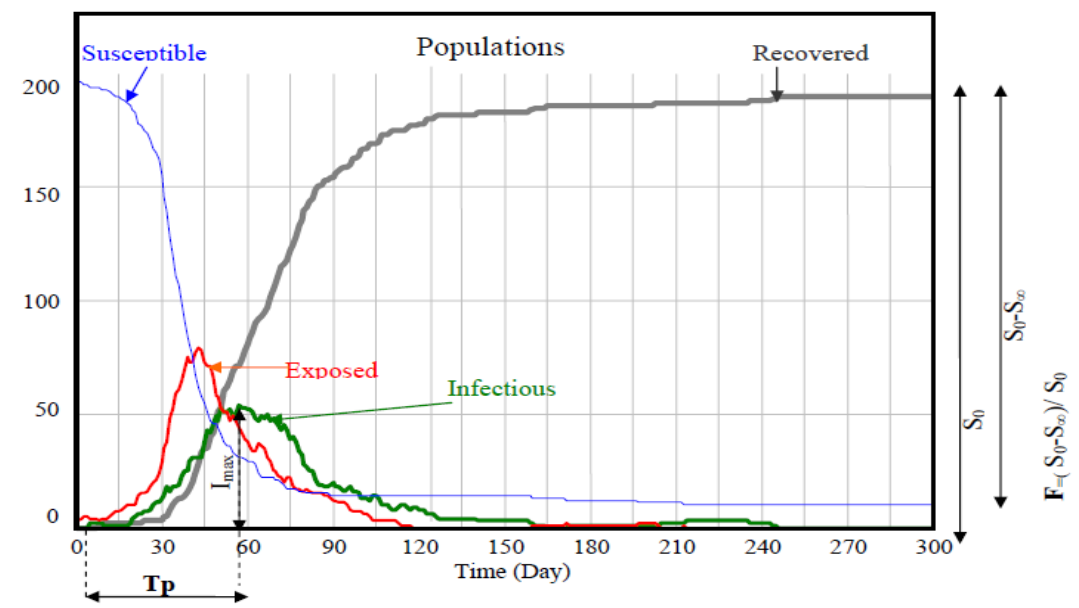

Figure 6: A sample simulation showing four discrete states and three output measures.

Source: Rahmandad (2004).

\section{Results of the SEIR epidemic simulation model for Mkole, Mkinga and Korongwe Villages}

Results of the SEIR epidemic simulation of scale-free contact networks in all three villages for both measles outbreak occurring at a single individual or multiple individuals show an increase in the number of recovered individuals at recovery rate of $1 / 7$, and a decrease in number of infected individuals at an infectivity rate of $1 / 8$ (Figures $7-8$ ) within an average period of 100 days. It is also important to note that in Mkole village the epidemic peaks later in measles outbreaks occurring at single individual, while it peaks sooner in measles outbreaks occurring at multiple individuals.

More importantly, two villages (Mkinga and Korongwe) have the same peak time for measles outbreak occurring at single individual and a difference of one day for measles outbreak occurring at multiple individuals. Specifically, Korongwe village has a difference of 5 days between these two measles outbreaks and Mkinga village has a difference of 4 days between these two measles outbreaks. This is unlike Mkole village where the difference is three weeks (21 days). A possible explanation may be the nature of these two predominantly fishing villages whose populations and average sizes of households and other locations such as schools are larger than that of Mkole Village.

Examining thoroughly results for the SEIR epidemic simulation model, one notices that an increase in the percentage of infected population immediately after the start of an epidemic is simply because of the nature of scale-free networks but also the fact that measles has a fairly high basic reproduction number; $\quad R_{0}$ is approximately $10-20$ (Sugihara and May 1990). After a brief interval of infectiousness, recovered individuals are immune at the long run and this tends to reproduce long-lasting 'interepidemic' oscillations, with a period of about 2 years, even in the simplest models. 


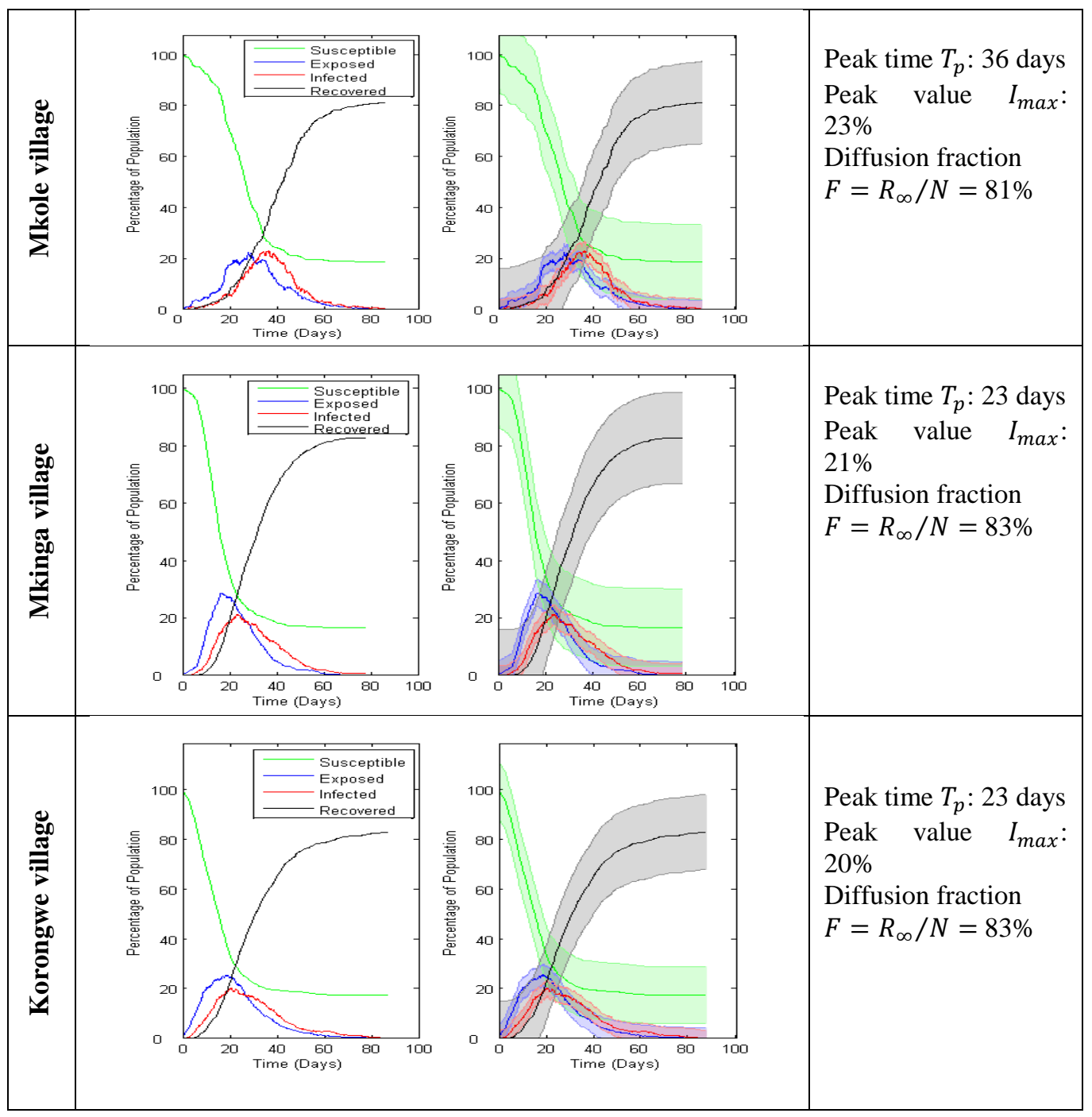

Figure 7: SEIR epidemic simulation model for Mkole, Mkinga and Korongwe Villages: Recovery rate $\tau=1 / 7$; Infectivity rate $\gamma=1 / 8 ; I_{0}=1$.

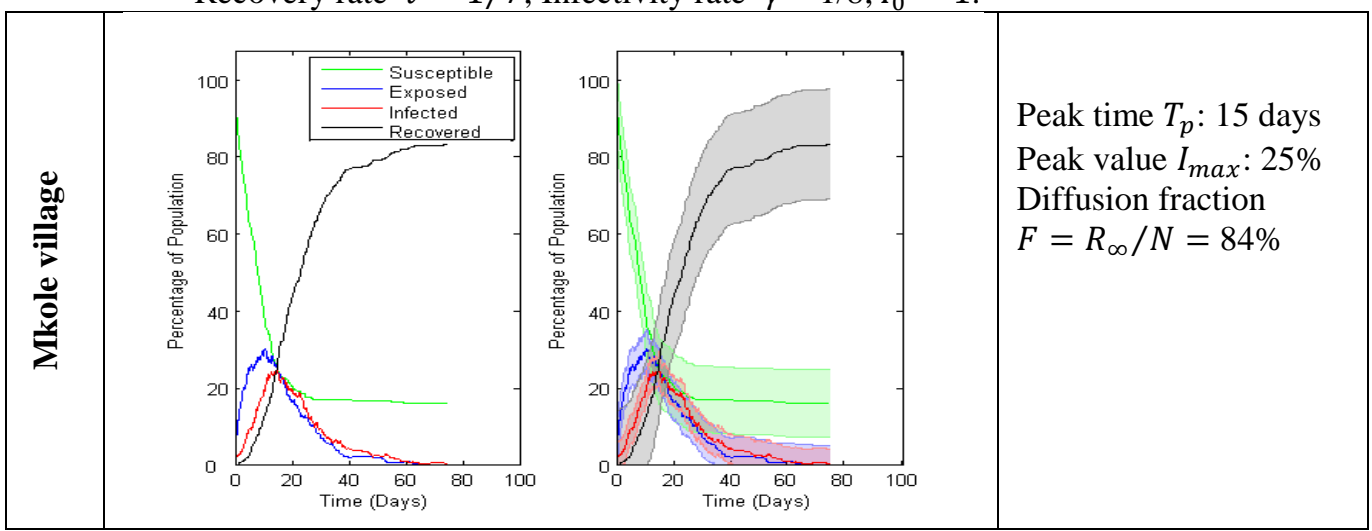




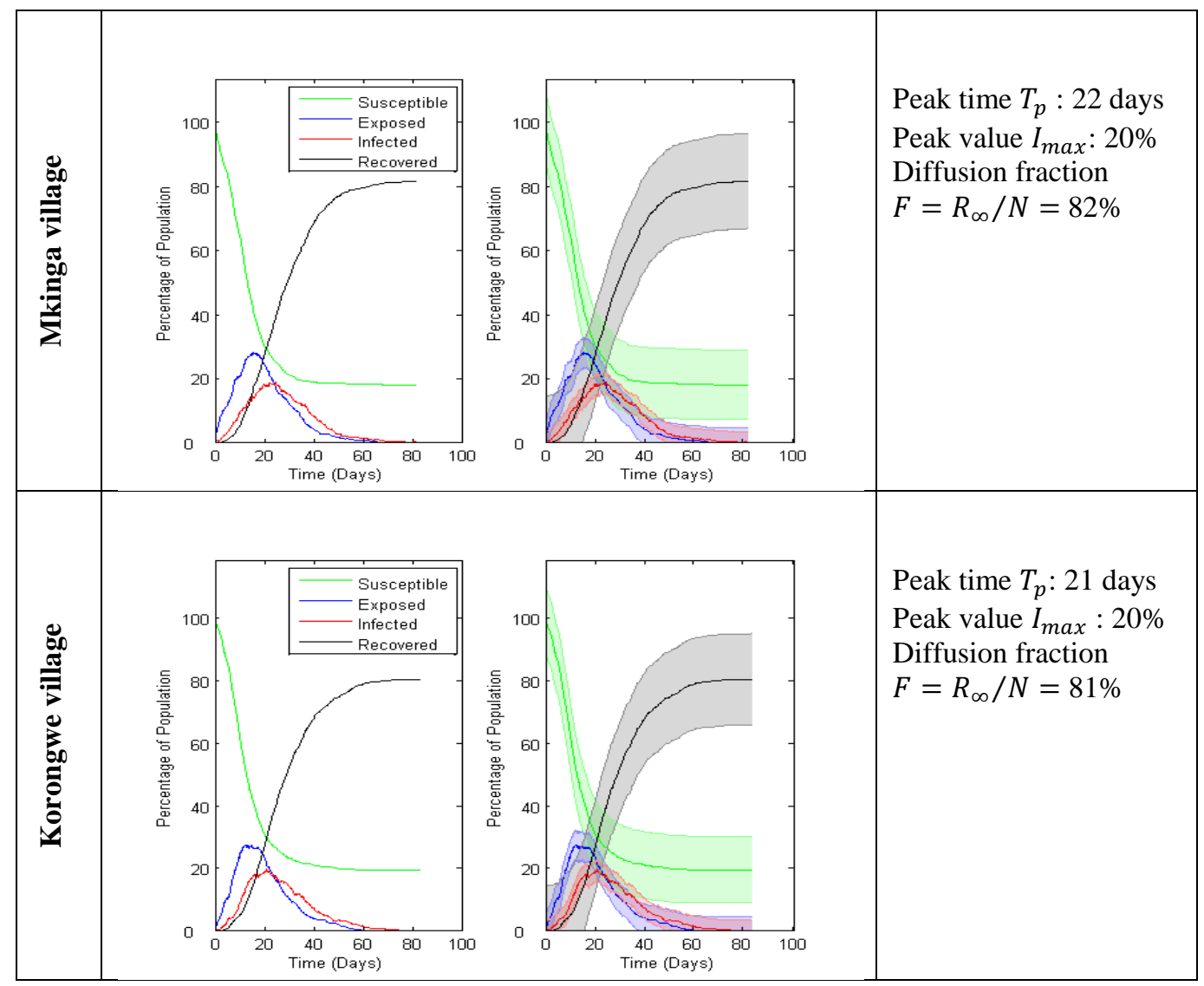

Figure 8: SEIR epidemic simulation model for Mkole, Mkinga and Korongwe villages: Recovery rate $\tau=1 / 7$; Infectivity rate $\gamma=1 / 8 ; I_{0}=5$.

\section{Model fitting}

To underscore the robustness of our model, we compare real data with the model simulation by fitting the epidemic data from Mkinga village obtained from the office of Nkasi District Medical Officer. This data is routinely collected by the respective office. Comparison of simulated and real (actual) data shows similar trends of curves irrespective of different rates of infections. This is an indication that our simulation is robust enough and a valid representation of the real situation, thereby providing confidence in our policy recommendations. Note that in Figure 9 the percentage of infected individuals did not rise significantly between the initial data points. Explanation behind this might be the fact that the 2009 outbreak in Nkasi District for instance, occurred in July. According to Tanzania's school calendar, this is the time of schools' holiday, thus measles transmission rate in schools as one of the contact points is very limited in July.

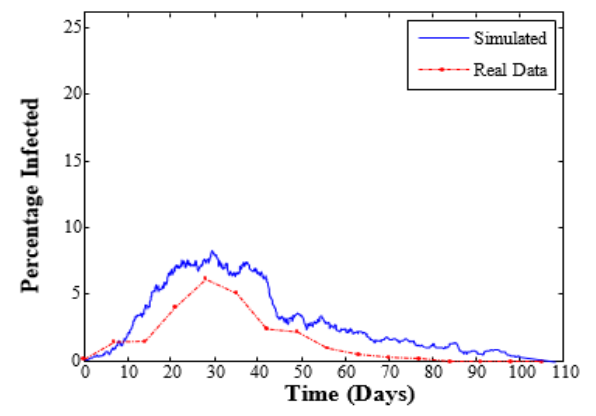

Figure 9: Mkinga Village real data and simulated curves: Recovery rate $\tau=1 / 5$; Infectivity rate $\gamma=1 / 10 ; I_{0}=1$. 


\section{The SEIR vaccination epidemic simulation}

This part presents results and discussion of a SEIR simulation model of vaccine intervention. Both mass vaccination and targeted vaccination strategies are presented and the optimal (improved) one is proposed. Our SEIR simulation model of vaccine interventions considers ages $\leq 6$ years to include children from 6 months up to 6 years inclusive; $<5$ years (from 6 months to 59 months); $\leq 15$ years (from 6 months up to 15 years inclusive) and $\leq 19$ years (from 6

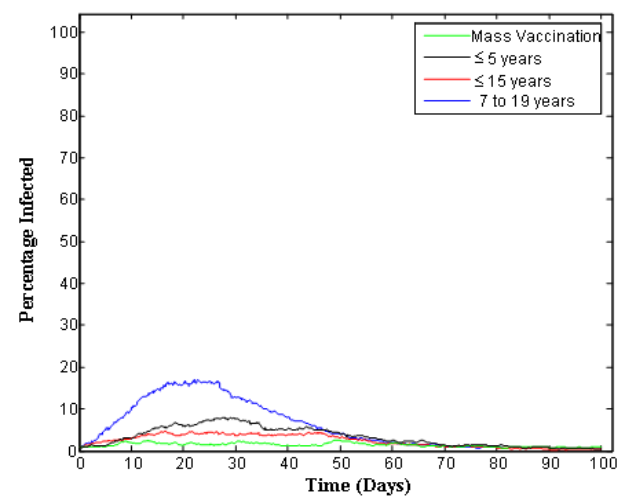

months up to 19 years inclusive). All simulations for both mass and targeted vaccination strategies were run with recovery rate $\tau=1 / 7$; infectivity rate $\gamma=1 / 8 ; I_{0}=5$; and vaccine efficacy $\varepsilon=0.85$. The graphical representations of these simulations are depicted in Figures 10-12 (The left graphic shows the SEIR vaccination simulation result of percentage for all infected individuals, the right graphic shows the percentage for recovered individuals).

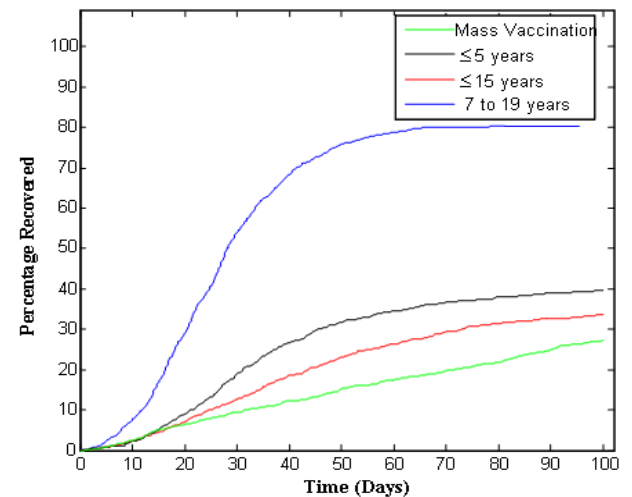

Figure 10: Korongwe Village results for vaccination strategies.
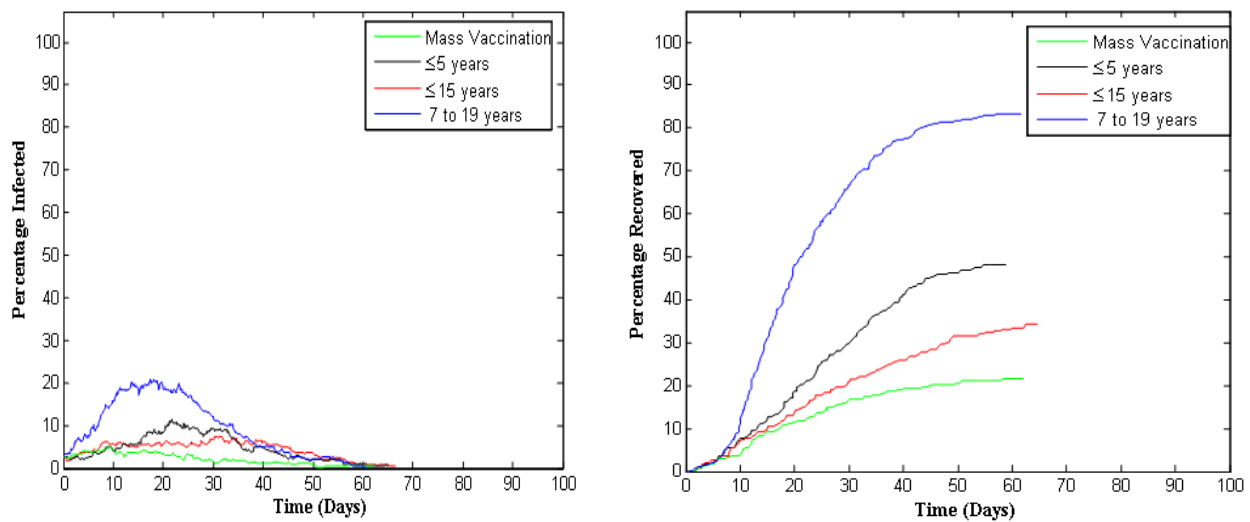

Figure 11: Mkole Village results for vaccination strategies. 

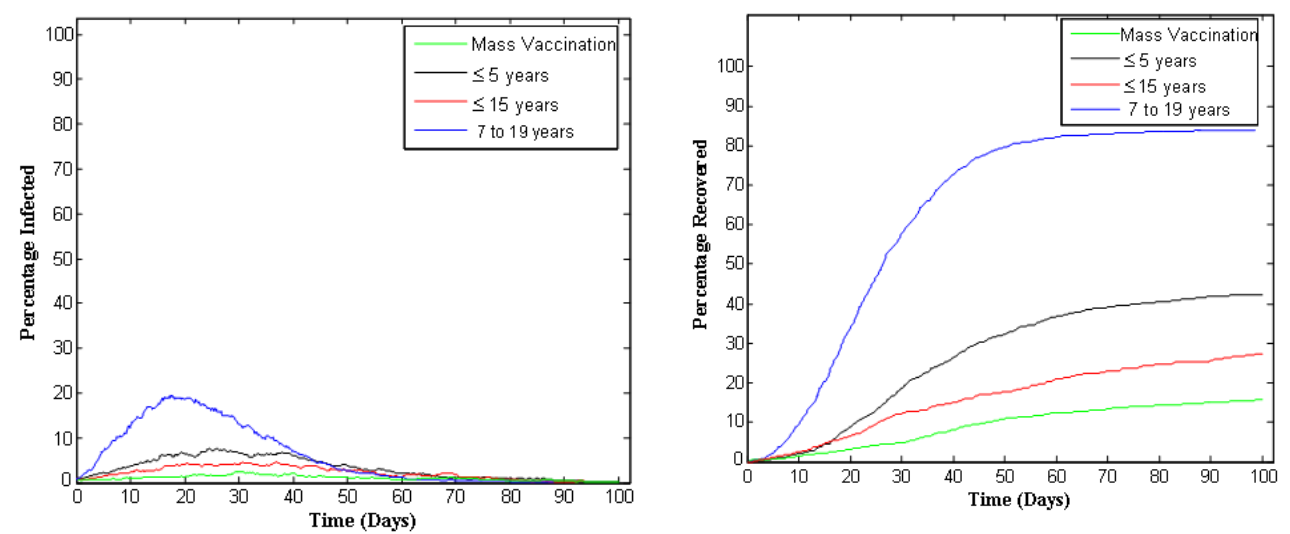

Figure 12: Mkinga Village results for vaccination strategies.

The overall results of the SEIR model of mass vaccination for all susceptible individuals in the three villages show that if measles vaccination is administered as soon as an outbreak occurs, it can raise immunization levels of individuals in populations. This aligns with WHO guidelines that in response to measles outbreaks in mortality-reduction settings, measles vaccine as a control measure should be administered before the natural end of the outbreak to help mitigate the number of cases and subsequent deaths (WHO 2009). Based on a late implementation of measles mass vaccination campaign in Maroua, Cameroon, Luquero et al. (2011) noted that time is important when conducting Outbreak Response Immunizations (ORIs) as its potential impact could have been higher with earlier implementation. Grais et al. (2007) also had similar observation from a measles outbreak in Niamey, Niger that timely implementation of ORI for children aged 6 months to 15 years could result in substantial numbers of cases averted as their epidemic curve showed a decrease of $50 \%$ cases beginning 2 weeks after ORI for the campaign targeting all children aged 6 to 59 months. However, because the vaccine efficacy is estimated to be approximately 85 percent, one may notice minimal transmissions in our mass vaccination results, which is also the case with a targeted vaccination strategy for children of 6 months to 15 years of age.
Low- and middle-income countries like Tanzania cannot easily afford costs associated with implementing mass vaccination targeting all age groups, our findings indicate that public health policy in Tanzania should emphasize on a targeted vaccination strategy. Gay (2004) noted that the use of a single dose of measles vaccine cannot achieve a high enough level of immunity for elimination and that a routine 2dose schedule can achieve high levels of immunity (98\% efficacy), though it will take many years to feed through all age groups because outbreaks often occur in the cohorts just too old to have received 2 doses. Thus, it will be necessary to maintain high 2-dose vaccination coverage (Fiebelkorn et al. 2017). It is within the rationale of this argument therefore, that our findings suggest more efforts to be employed on targeted vaccination, particularly of older age groups in Tanzania who appear to be missed or to be born before the second dose schedule, for the effective control of the spread of measles. This also agrees with our third assumption that vaccination provides partial protection to vaccinated individuals but if administered at regular intervals it provides an effective and long-term protection.

Based on these results, vaccination strategies targeting both children $\leq 5$ years and those $\leq 15$ years might be recommended. These reflect the common practice recommended by The Sphere Project (2004) for humanitarian emergencies, which advocates vaccinating against measles in all 
children between the ages 6 months and $<15$ years old. Also, our recommendation is in line with that of Kamugisha et al. (2003) who reported $31 \%$ of measles cases among children between 6 months and 15 years during an outbreak among Burundian refugee camps in Tanzania. This recommendation is further supported by Grais et al. (2011) that a non-selective response targeting children 6 months to 5 years, initiated between one and five months after cases were first reported reduced cases and deaths, but it would have been more effective to target a wider age range to halt transmission.

\section{Conclusion}

The results indicate that the spread of measles largely depends on the social contact network rates of infected individuals in the community. A similar methodology employed by this work could readily be applied to more complex epidemic models in other measles outbreak regions. Despite the success of mass vaccination campaigns, our results emphasize a need for a targeted vaccination strategy, especially given Tanzania limited economy to cater for a wide age range vaccination. This paper adds to the measles vaccination theory and its implementation due to the usefulness of social contact network epidemiological modeling in determining the age range for targeted vaccination, especially by comparing the susceptibility profile of the population against the susceptibility targets. By vaccinating children of 6 months to 15 years of age, but also equally vaccinating older age groups who were born before 1957 or missed the second dose schedule may prove an effective vaccination. This paper offers an opportunity of designing effective measles vaccination programs in Tanzania and other developing countries while addressing potential implications of each intervention strategy.

While efforts were made to formulate and rigorously analyze epidemiological models of measles spread, this paper does not purport to be exhaustive. For the model parameters and assumptions, we searched a wide range of literature and Tanzania-specific reports such as the Country-Wide Time Use survey in
Tanzania (2006), the Value of Time in Least Developed Countries, and the Department for International Development (2005). However, given the nature of heterogeneously mixed populations of Mkole, Mkinga and Korongwe villages, assumptions from other geographical settings may not necessarily be realistic in these villages.

Conflict of Interest: No conflict of interest.

\section{References}

Adhvaryu A and Nyshadham A 2011 Schooling, Child Labor and the Returns to Healthcare in Tanzania, Yale Labor/Public Finance Lunch and the IZA Workshop on Child Labor.

Advanced Immunization Management 2009 WHO's Measles Strategic Planning (MSP).

https://extranet.who.int/aim_elearning/en/ measles/tool.pdf Accessed July, $2^{\text {nd }} 2011$.

Anderson RM and May RM 1991 Infectious Diseases of Humans: Dynamics and Control. Oxford, UK: Oxford University Press.

Andradóttir S, Chiu W, Goldsman D, Lee ML, Tsui K, Sander B, Fisman DN and Nizam A 2011 Reactive Strategies for Containing Developing Outbreaks of Pandemic Influenza. BMC Public Health 11(1):1-15.

AndvigJ, Canagarajah S and Kielland A 2001 Issues in Child Labor in Africa, Working Paper Series Human Development Sector Africa Region, World Bank.

Barabàsi AL and Albert R 1999 Emergence of Scaling in Random Networks. Science 286: 509-512.

Berhe HW and Makinde OD 2020 Computational modelling and optimal control of measles epidemic in human population. Biosystems 190: 104102.

Burke K and Beegle K 2004 Why children aren't attending school: the case of northwestern Tanzania. J. Afr. Econ. 13(2): 333-355.

Chen FH and Cottrell A 2009 Dynamic equilibria in an epidemic model with voluntary vaccinations. J. Biol. Dynamics 3(4): 357-375. 
Chowell-Puente G and Sanchez F 2001 Disease dynamics on small-world and other networks. Cornell University, Dept. of Biometrics Technical Report BU-1588M.

Del Valle S, Hyman J, Hethcote $\mathrm{H}$ and Eubank S 2007 Mixing patterns between age groups in social networks. Social Networks29: 539-554.

Department for International Development 2005 The value of time in least developed countries: The African Studies (R8307) Final Report. Project Report Contract No. R8307. I.T. Transport Ltd., Ardington, Oxfordshire, United Kingdom.

Easley D and Kleinberg J 2010 Networks, Crowds, and Markets: Reasoning about a Highly Connected World. Cornell, NY: Cambridge University Press.

Fiebelkorn AP, Redd SB, Gastañaduy PA, Clemmons N, Rota PA, Rota JS, Bellini WJ and Wallace GS 2017 A comparison of post-elimination measles epidemiology in the United States, 2009-2014 versus 2001-2008. J. Pediatr. Infect. Dis. Soc. 6(1): 40-48.

Gay NJ 2004 The theory of measles elimination: Implications for the design of elimination strategies. J. Infect. Dis. 189(1): S27-35.

Goldrick BA 2019 The alarming global increase in measles. Am. J. Nurs. 119(6): 14.

Goodson J, Wiesen E, Perry R, Mach O, Kitambi M,Kibona M, Luman $\mathrm{E}$ and Cairns K 2009 Impact of measles outbreak response vaccination campaign in Dar es Salaam, Tanzania. Vaccine 27: 5870-5874.

Grais R, Dubray C, Gerstl S, Guthmann J, Djibo A, Nargaye K, Coker J, Alberti K, Cochet A, Ihekweazu C, Nathan N, Payne L,Porten K, Sauvageot D, Schimmer B, Fermon F, Burny M, Hersh B and Guerin P 2007 Unacceptably high mortality related to measles epidemics in Niger, Nigeria, and Chad. PLoS Med. 4(1): e16.

Grais RF, Strebel P, Mala P, Watson J, Nandy R and Michelle G 2011 Measles vaccination in humanitarian emergencies: a review of recent practice. Conflict Health 5: 21.

Haber MJ, Shay DK, Davis XM, Patel R, Jin $\mathrm{X}$, Weintraub E, Orenstein $\mathrm{E}$ and Thompson W 2007 Effectiveness of interventions to reduce contact rates during a simulated influenza pandemic. Emerg. Infect. Dis. 13(4): 581-589.

Kamugisha C, Cairns K and Akim C 2003 An outbreak of measles in Tanzanian refugee camps. J. Infect. Dis. 187(1): 58-62.

Kondylis F and Manacorda M 2006 School Proximity and Child Labor Evidence from Rural Tanzania, Nuffield Foundation (New Career Development Fellowship in the Social Sciences) and ESRC.

Luquero FJ, Pham-Orsetti H, Cummings DAT, Ngaunji PE, Marcelino N, Fermon F, Ngoe N, Sosler S, Strebel P and Grai RF 2011 A long-lasting measles epidemic in Maroua, Cameroon 2008-2009: Mass vaccination as response to the epidemic. J. Infect. Dis. 204: S243-S251.

Lyamuya E, Matee M, Aaby P and Scheutz F 1999 Serum levels of measles IgG antibody in children under 5 years in Dar es Salaam, Tanzania. Ann. Trop. Paeditr. 19(2): 175-183.

Mandara M and Remme J 1983 Current Measles Control in Tanzania. Rev. Infect. Dis. 5(3): 554-557.

Martín G, Marinescu M, Singh DE and Carretero J 2011 Leveraging social networks for understanding the evolution of epidemics. BMC Systems Biology 5(3): S14.

May RM and Lloyd AL 2001 Infection dynamics on scale free networks. Phys. Rev. E Stat. Nonlin. Soft. Matter Phys. 64: 066112.

Meyers LA, Newman ME, Martin M and Schrag S 2003 Applying network theory to epidemics: control measures for Mycoplasma pneumoniae outbreaks. Emerg. Inf. Dis. 9: 204-210.

Meyers LA, Pourbohloul B, Newman M, Skowronski D and Brunham R 2005 Network theory and SARS: Predicting outbreak diversity. J. Theor. Biol. 232: 71-81. 
Mnyika SK and Akim C 2005 Epidemiology of measles in Tanzania: A hospital-based survey of measles morbidity and mortality. East Afr. J. Public Health 2(2): 24-27.

Newman MEJ 2002 Spread of epidemic disease on networks. Phys. Rev. E66: 016128.

NUFU Project 2006 Country-wide time use by gender and advanced analysis of census data: Main Time Use Survey Report. University of Dar es Salaam and University of Troms $\varnothing$, Project ID: PRO 62/2003.

Orenstein WA, Bernier RH, Dondero TJ, Hinman AR, Marks JS, Bart KJ and Sirotkin B 1985 Field evaluation of vaccine efficacy. Bull. W. H. O. 63: 10551068.

Patel MK, Goodson JL and Alexander Jr JP, Kretsinger K, Sodha SV, Steulet C, Gacic-Dobo M, Rota PA, McFarland J, Menning L and Mulders MN 2020 Progress toward regional measles elimination-worldwide, 2000-2019. MMWR Morb. Mortal Wkly. Rep. 69(45): 1700-1705.

Pourbohloul B, Meyers L, Skowronski D, Krajden M, Patrick D, Robert C and Brunham R 2005 modeling control Strategies of Respiratory Pathogens. Emerg. Infect. Dis. 11(8): 1249-1256.

Rahmandad H 2004 Heterogeneity and network structure in the dynamics of contagion: Comparing agent-based and differential equation models, in the $22^{\text {nd }}$ International Conference of the System Dynamics Society. July 25-29, 2004. Oxford, England: System Dynamics Society. (CD \& Online, pp.1-32).

Sowole SO, Ibrahim A, Sangare Dand Lukman AO 2020 Mathematical model for measles disease with control on the susceptible and exposed compartments, Open J. Math. Sci. 4(1): 60-75.

Sugihara G and May R 1990 Nonlinear fore casting as a way of distinguishing chaos from measurement error in a data series. Nature 344:734-741.

Talley L and Salama P 2003 Assessing field vaccine efficacy for measles in famineaffected rural Ethiopia. Am. J. Trop. Med. Hyg. 68(5): 545-546.

The Sphere Project 2004 The Sphere Handbook: Humanitarian Charter and Minimum Standards in Disaster Response. Geneva: The Sphere Project.

United Republic of Tanzania 2002 Population and Housing Census, NBS, 2003.

WHO (World Health Organization) 2008 Measles reported cases. http://wwwwho.int/immunization_monito ring/en/globalsummary/timeseries/tsincid encemea.html. Accessed $2^{\text {nd }}$ October 2020.

WHO 2018 Measles cases spike globally due to gaps in vaccination coverage.News Release.Geneva/Atlanta/New York.

WHO 2019 Measles-Global situation. https://www.who.int/csr/don/26november-2019-measles global_situation/en/Accessed $8^{\text {th }}$ February 2021.

WHO 2021 Distribution of cases by country, year and month, https://www.who.int/immunization/monit oring_surveillance/burden/vpd/surveillanc e type/active/measles_monthlydata/en/, Accessed $20^{\text {th }}$ June 2021.

WHO 2020 Worldwide measles deaths climb $50 \%$ from 2016 to 2019 claiming over 207,500 lives in 2019, www.who.int/news/item/12-11-2020worldwide-measles-deaths-climb-50from-2016-to-2019-claiming-over-207500-lives-in-2019. Accessed $11^{\text {th }}$ January 2021.

WHO 2009 Response to Measles Outbreaks in Measles Mortality Reduction Settings. Geneva, Switzerland: World Health Organization, Department of Immunizations, Vaccines and Biologicals; WHO publication WHO/IVB/09/03. 